\title{
Vulnerability Research and Mapping of Campus Network
}

\author{
Justinus Andjarwirawan \\ Petra Christian University/Informatics Department, Surabaya, Indonesia \\ Email: justin@petra.ac.id \\ Agustinus Noertjahyana and Devi C. Angi \\ Petra Christian University/Informatics Department, Surabaya, Indonesia \\ Email: agust@petra.ac.id,m26411163@john.petra.ac.id
}

\begin{abstract}
Vulnerability of computer systems in campuswide network has been an issue for years, since networks were open to allow anonymous access. It will take many studies of computer security to protect. A vulnerability research and mapping of a network is a step to address the issue, as well as minimizing security breach in the future.

Evaluation of a network security can be done by many tools available and also guidelines based on CEH (Certified Ethical Hacker) module and Acunetix for web specific security.

With CEH and Acunetix guidelines, the evaluation shows many common security weaknesses and therefor this evaluation leads to security recommendations based on the weaknesses and security holes found.
\end{abstract}

Index Terms - vulnerability, penetration testing, vulnerability scanning, certified ethical hacker, acunetix

\section{INTRODUCTION}

Network security evaluation is done inside Petra Christian University, Surabaya, Indonesia, as the target. The chosen test object has a very common university network configuration. Common configuration also delivers common attacks such as computer virus, trojan, backdoors, spams, as well as cross-site scripting and SQL injection.

Universities today still manage their own web servers, mail servers and databases. The focus of monitoring intrusions are on these servers, besides the routers and firewalls.

Penetration test or pentest is one method to evaluate a computer network security parameter. Most reports of the penetration test are used to define protections against possible future attack.

\section{A. Problem Statements}

Monitoring campus-wide computer network and servers. The first step to evaluate this wide network is collecting all information about the existing network and active servers in the area.

Expected solutions to secure a campus-wide network are based on the evaluation. Mapping is given after a full scan of the entire network. A report of the whole scanning, penetration test and test tools results is given at the end of the test. CEH (Certified Ethical Hacker) by EC-Council will be the guideline to analyze and implement a security policy, as suggested (K. Graves, 2010)[5].

Acunetix is a software as a tool to analyze vulnerabilites of web sites and web based applications. Cross-site Scripting (XSS) and SQL injection are the top security vulnerabilities found.

ints after.

\section{B. Methodology}

Surveys of the existing Local Area Networks (LANs), servers, infrastructure are done both by interviews and own scanning. Accessing to the campus network is available through wireless network, wired network, and also the Internet.

Filter and limitation of the network access through a router or firewall are easily detected by doing a port scanning with nmap tool. A firewall test must be done both ways, from inside the campus network to the Internet and from the Internet to the demilitarized zone (DMZ) of campus network.

\section{THEORIES}

\section{A. Network Security}

There is no network that is capable of anti tapping nor computer network that is completely secure. The nature of a computer network is for communication. Each communication can fall into the hands of others and can be misused. Security systems help secure the network without blocking the user and puts anticipation when the network successfully penetrated. In addition, it is important to make sure that the users in the network have sufficient knowledge about the safety and that they accept and understand how the security plans are made.

There are two main elements of forming a secure network, they are:

1. Firewall, both physical and virtual, which is placed between the device and network services used and the people who may misbehave. 
2. The security plan, which will be implemented together with the users to keep the system from penetration from outside.

In addition to those described above, network security can be aimed for information system owners that can keep their information system from compromised by others, which in turn can damage the system.

The type of intruder may include:

- The Curious: this type of intruder is basically interested in finding the type of system and data owned by a person or company.

- The Malicious: type of intruder is trying to ruin someone else's system or modify a webpage.

- The High-Profile Intruder: this type is trying to use someone else's system to gain popularity and fame.

- The Competition: this type of intruder is interested in what data you have in the system of organization or others.

Computer security is one important aspect of an information system. Meanwhile, a network itself can also be secured from attacks. In terms of the types of existing network security, they are: Confidentiality, Integrity, Availability, Non-repudiation, Authentication and Accountability.

\section{B. Hacking}

Hacking is a common term that is usually negative, but in the definition itself hacking is a way of someone who manipulates things to perform better or useful for another purpose. A negative person doing a bad hacking is known as a cracker, or those who do cracking a system.

A computer hacker term may mean either bad or good depending on the purpose of hacking. A hacker may damage confidential information, steal confidential information, and also modify them.

But a hacker may do good things for good purposes, that they were called white hat hackers. Also a good hacker term is used for the certification such as CEH.

\section{Penetration Test}

The purpose of penetration testing is to find vulnerabilities of a system. There are two types of testing, they are: external testing and internal testing.

External testing is to test all available information and access that is available to public or without any kind of authentication.

Internal testing is to recognize the number of network access points internally.

The analysis done in this research to the objective campus is by using the external testing.

The three methods of penetration testing are: Passive, Active and Aggressive. Passive test will test inside web applications, logins and configurations. Active test will do input manipulations, possess access rights and test all known vulnerabilities. Aggressive test will do the vulnerability exploits, reverse engineer a software or system, putting a backdoor, steal codes and manipulate finance related information.

The campus object test is done with the passive type of penetration test.
The penetration phases are: discovery, enumeration, vulnerability mapping, exploitation, as also found in many campus wide penetration tests[3][4], and finally report generation.

\section{ANALYSIS}

The purpose of this research is to find weaknesses, that is the vulnerabilities of the network and systems, then also to deliver solutions to secure those vulnerabilities.

The tools used for the test are penetration tools softwares that are suggested by CEH.

The first tool is for footprinting, using Angry IP Scanner, and then scanning with Acunetix, and finally enumeration with Softperfect Network Scanner. Some tools that are suggested by $\mathrm{CEH}$ are paid commercial applications. The rest are open source applications.

According to $\mathrm{CEH}, 90 \%$ of the time a hacker spends their time is to collect information. The rest are to take over the target.

Footprinting which is the first thing to do as a hacker is to define the target by doing port scanning to all available IP addresses of the target network, to find available services and active hosts.

Footprinting the campus network finds information:

- target IP addresses

- live services: web (http \& https), ftp, ssh

- host names

- application version (e.g. web server version)

The Acunetix tool delivers a report that counts the numbers of vulnerabilities and the level of the security vulnerability, from low, medium, to high; as in Microsoft[1]. Table I shows major vulnerabilites found in the testing.

TABLE I. VULNERABILITY SUMMARY

\begin{tabular}{|c|c|c|c|c|}
\hline \multicolumn{5}{|c|}{ Description } \\
\hline No. & Attacks & Vulnerability & $\begin{array}{c}\text { Total } \\
\text { alerts }\end{array}$ & Level \\
\hline & $\begin{array}{c}\text { Cross Site } \\
\text { Scripting } \\
\text { (XSS) }\end{array}$ & $\begin{array}{c}\text { Cross site } \\
\text { scripting } \\
\text { (verified) }\end{array}$ & 12 & High \\
\cline { 3 - 5 } 2 & $\begin{array}{c}\text { jQuery cross } \\
\text { site scripting }\end{array}$ & 8 & High \\
\hline 3 & $\begin{array}{c}\text { SQL } \\
\text { Injection }\end{array}$ & $\begin{array}{c}\text { Blind SQL } \\
\text { Injection }\end{array}$ & 7 & High \\
\hline $\begin{array}{c}\text { CSRF } \\
\text { Cross Site } \\
\text { Request } \\
\text { Forgery) } \\
\text { protection }\end{array}$ & $\begin{array}{c}\text { HTML form } \\
\text { without } \\
\text { CSRF } \\
\text { protection }\end{array}$ & 18 & Medium \\
\hline
\end{tabular}

\section{CONCLUSION}

Port scannings were not completely tested on all available ports, which may miss a few known services that changed their default ports. 
Many web sites and web applications are found to be vulnerable to Distributed Denial of Service (DDoS).

Old versions of web servers are also spotted. Many web applications are found to have using old versions and deprecated functions which could cause a Cross-Site Scripting and SQL injection attacks. These are the most vulnerabilities found commonly in other organizations[2].

The suggestions are to always keep an up to date versions of operating systems and applications. Also to do periodic tests. It is also suggested to classify port penetration attempts and in percentage values[1] and weights, therefore administrators will have priorities to anticipate future attacks based on the most probable attempts.

\section{ACKNOWLEDGMENT}

The author wish to thank the ICCCV committees for accepting this paper, and also Agustinus Noertjahyana and Devi C. Angi for supporting the research.

\section{REFERENCES}

[1] Yeu-Pon Lai, Po-Lun Hsia, "Using the vulnerability information of computer systems to improve the network security", Computer Communications Volume 30, issue 9, Elsevier, 30 June 2007, pp. 2032-2047.
[2] Mvungi, N.H., Mfinanga, D.A., Mwinyiwiwa, B.M.M., "Intrussion detection by penetration test in an organization network", in Adaptive Science \& Technology, IEEE, Jan 2009, pp. 226-231.

[3] Zulazeze Sahri, Muhd Eizan Shafiq Abd Aziz, Khairul Ikhwan Zolkefley, Roslan Sadjirin, Mohd Ikhsan Md Raus, "Implementing IT Security Penetration Testing in Higher Education Institute", in Australian Journal of Basic and Applied Sciences, ISSN: 19918178, 2014, pp. 67-72.

[4] Arya Sedigh, Kapilan Radhakrishnana, Carlene E-A Campbella, Dhananjay Singh, "Trust Evaluation of the Current Security Measures Against Key Network Attacks", MAGNT Research Report, ISSN 1444-8939, 2014.

[5] Kimberly Graves, "CEH Certified Ethical Hacker Study Guide", Sybex, 2010.

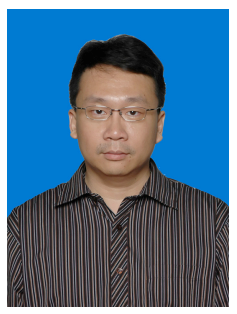

Justinus Andjarwirawan is a lecturer in the Informatics Engineering department of Petra Christian University. It is based in Surabaya city, Indonesia. Born in Central Java, Indonesia in 1972.

$\mathrm{He}$ has a bachelor degree of Electrical Engineering from Petra Christian University, Indonesia, and a master degree in Computer Science from Asian Institute of Technology, Thailand. His current interest and teaching subjects are Open Source Programming, Mobile Programming, and Web Programming. Research interest in subjects of computer networking, user experience, user interface, human-computer interaction, web technology, and mobile applications. 